\title{
Optimization of the concrete through the addition of nanosilice, using aggregates of the cantera de Añashuayco de Arequipa

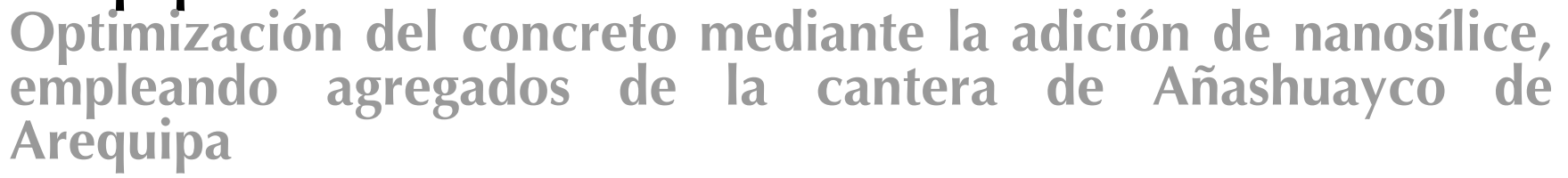

Caballero Arredondo, P.W. ${ }^{1}$ *, Damiani Lazo, C. A. ${ }^{*}$, Ruiz Pico, Á.A. ${ }^{* *}$

* Universidad Nacional de San Agustín de Arequipa

** Universidad Santo Toribio de Mogrovejo

Fecha de Recepción: 13/07/2020

Fecha de Aceptación: 10/10/2020

PAG 71-87

\begin{abstract}
The present research work is carried out using the experimental study to evaluate the concrete made with Aggregates of the Quarry with Addition of Nanosilica containing micro-fine nanoparticles of amorphous silica dioxide (Si O). Nanosilica has pozzolanic properties that, upon contact with water, reacts with hydrated cement to form more particles of $\mathrm{C}-\mathrm{S}-\mathrm{H}$ (gel), which reduces porosity by partially filling the pores to refine the structure and therefore gives us greater compressive strength. The recommendation of this study is to implement low-cost, low-rise housing construction.

The microstructure properties of the cement paste are improved with the addition of Nanosilica. Nanoparticles have high specific surface areas, and their surfaces are very active; an intense pozzolanic reaction between Nanosilica and $\mathrm{CH}$, would accelerate the pozzolanic reactions between SIO and water molecules. Therefore, the smaller the pore volume, the higher the compressive strength and water absorption capacity of Nanosilica-modified pastes results.

The volume of the pores was reduced along with the increase of the Nanosilica dose. The cement and sand mixture without Nanosilica after 7 days of curing, shows a porous structure.
\end{abstract}

Keywords: Nanosilica, strength, compression, permeability

Resumen

El presente trabajo de investigación se desarrolla haciendo uso del estudio experimental para evaluar el concreto elaborado con agregados de la cantera de Añashuayco (Perú) con adición de Nanosílice que contiene nanopartículas micro finas de dióxido de sílice amorfa (Si O).

La Nanosílice posee propiedades puzolanicas que al contacto con agua reacciona con el cemento hidratado para formar más partículas de C-S-H (GEL), el cual reduce la porosidad rellenado parcialmente los poros para refinar la estructura y por consiguiente nos da una mayor resistencia a la compresión. Las recomendaciones de este estudio es implementar la construcción de viviendas de baja altura y bajo costo.

Las propiedades de microestructura de la pasta de cemento se mejoran con la adición de nanosílice. Las nanopartículas tienen áreas superficiales especificas elevadas, y sus superficies son muy activas; una reacción puzolanica intensa entre la nanosílice y el $\mathrm{CH}$, acelerarían las reacciones puzolanas entre SIO y moléculas de agua. Por lo tanto, a menor volumen de poros, resulta en una mayor resistencia a la comprensión y una capacidad de absorción de agua de las pastas modificadas con nanosílice.

El volumen de los poros se redujo junto con el aumento de la dosis de Nanosílice. La mezcla de cemento y arena sin nanosílice después de 7 días de curado, muestra una estructura porosa.

Palabras clave: Nanosílice, resistencia, compresión, permeabilidad

\section{Introducción}

En la última década Arequipa viene experimentando un crecimiento acelerado de su expansión urbana, así como de su infraestructura y otras vinculadas con la industria de la construcción.

Los concretos de alto desempeño que para el resto de la presente investigación le denominaremos CAD, están elaborados esencialmente por los mismos materiales que un concreto convencional. El objetivo de esta investigación es mejorar la calidad de un concreto con agregado natural de la cantera Añashuayco (Figura 1)), adicionando Nanosílice, con esto se pretende mejorar las propiedades del concreto, tomando en cuenta lo señalado por la NTP, para luego comparar y determinar la influencia de la adición de la Nanosílice en la resistencia final del concreto. Se contribuirá al mayor conocimiento en la materia y constituirá un aporte a la comunidad. Se dispondrá de un nuevo material ecológico, que implemente la Política Publica medio ambiental en Perú, empleando recursos propios de la zona, más económicos y construcciones más seguras.

\footnotetext{
1 Autor de correspondencia:

Universidad Nacional de San Agustín de Arequipa - Arequipa,

PERÚ

E-mail: $X X X X X X X X X$
} 


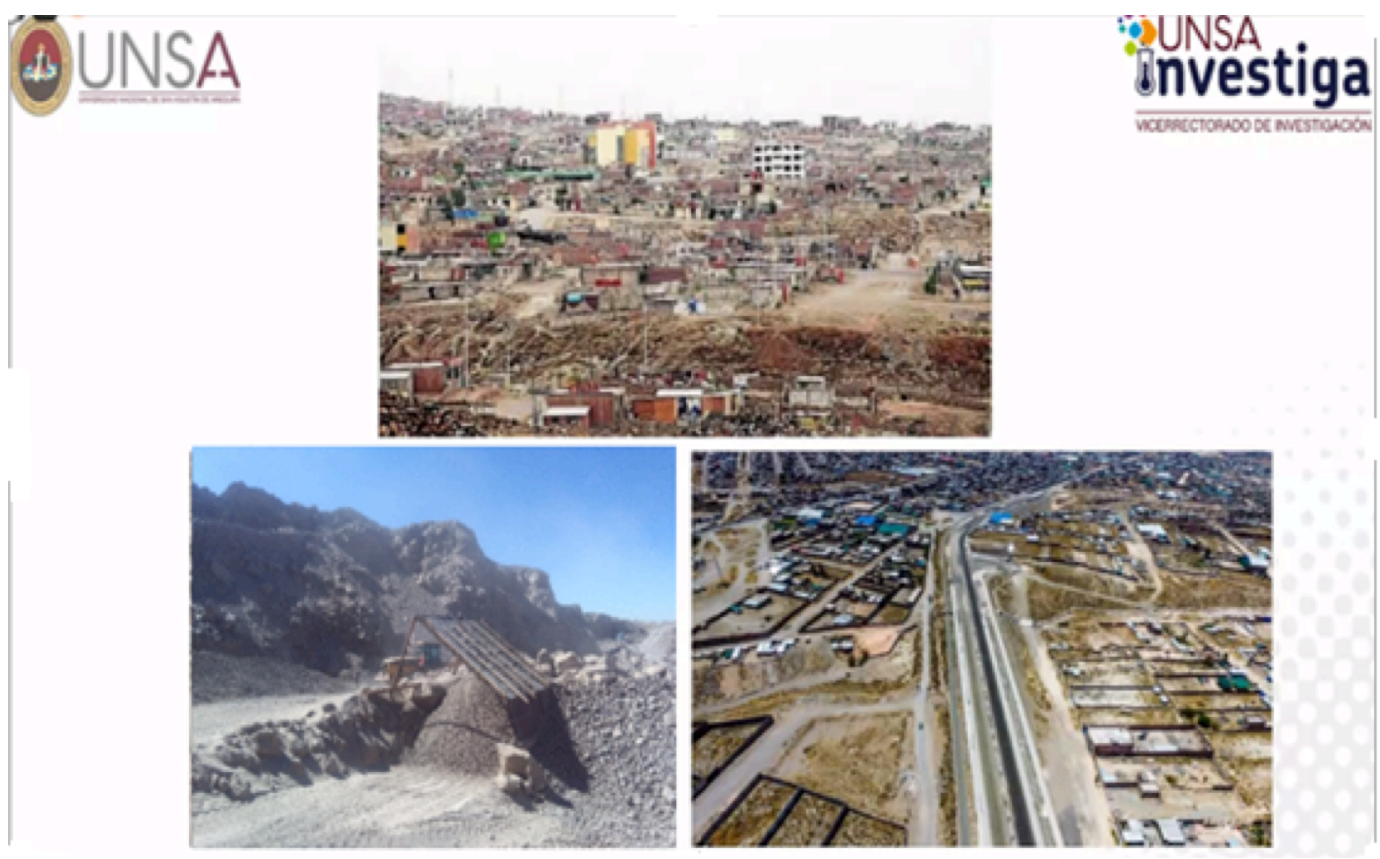

Figura 1. Agregado Natural de la Cantera de Añashuayco. Fuente: (Infraestructura Vial, Noticias Arequipa)

La Nanosílice debido a su alto contenido de Sílice $\left(\mathrm{SiO}_{2}\right)$, y a la finura de sus partículas incrementa la resistencia a la compresión del concreto, además de su fabricación amigable con el medio ambiente.

Según las investigaciones del autor (Fuentes at al., 2014) para obtener un concreto de alto desempeño (CAD) que supere la resistencia a la compresión de un concreto patrón convencional se requiere: (1) un material cementante que presente partículas menores a $0.5 \mu \mathrm{m}$, elevados contenidos de $\mathrm{SiO}_{2}$ y el uso de aditivos reductores de agua. Tal es el caso de la adición de Nanosílice en el concreto con agregados de Añashuayco. Este material con alto contenido químico de sílice $\mathrm{SiO}_{2}$ y finura incrementa la resistencia a compresión del concreto.

La nanosílice reacciona con portlandita para producir gel C-S-H adicional, por esta razón, la adhesión de nanosílice refino los poros y disminuyo el volumen de poros de las pastas. (Huang et al., 2021). También se reveló que la adición de nanosílice refinó la estructura de poros del hormigón y mejoro la resistencia a la penetración de cloruros del hormigón. (Zhang et al., 2011); la porosidad y el diámetro de los poros disminuyen con la inclusión de nanosílice. (Lim et al., 2018)

En otros estudios se puede observar que la incorporación de nanosílice en el suelo cementado incrementa la intensidad de hidróxido de calcio $(\mathrm{CH})$. El incremento en la densidad seca máxima de la arena cementada mezclada con nanosílice, puede demostrarse, por el hecho de que el vacío dentro del agregado, se vuelve ocupado por nanopartículas de nanosílice en el suelo cementado.(Choobbasti y Kutanaei, 2017).El empleo de nanosílice puede acortar el gasto de cemento evitando que libere $\mathrm{CO}_{2}$. Los efectos de la Nanosílice sobre el concreto son comparables, debido a su tamaño ultrafino y alta reactividad química, su rendimiento es mucho mejor con una menor cantidad de aditivo requerido. (Varghese et al.,2019) (Lincy et al., 2018)

Podemos decir que la mejora en el concreto, se atribuye a la aceleración de la hidratación del cemento, la rápida reacción puzolanica, así como el empaque optimizado de las partículas en la matriz del cemento. (Wang et al., 2018) (Kotsay, G., 2017) (El-Feky et al., 2019), lo que produce una alta probabilidad de segregación en el concreto. También se puede utilizar una dosis mucho menor de nanosílice para producir un hormigón ligero con un rendimiento mecánico comparable o mejor que uno que incorpora humo de sílice. (Sikora et al., 2020); aunque exceder la dosis optima de nanosílice resulto en una disminución en el efecto beneficioso de esta mezcla sobre la porosidad accesible al agua, (Meng et al., 2020) (Quercia et al., 2014) (Tobón et al., 2018)

La nanosílice mejora las características de la zona de transición y fortalece la unión entre el agregado y la pasta de cemento; por lo tanto, su adición dificulta en cierta medida el movimiento del agua en el concreto. (Du y 
Pang, 2019); por otro lado, la incorporación de nanosílice en dosis más pequeñas produce hormigones livianos con una microestructura mucho más robusta y mejores propiedades mecánicas y de transporte. (Sikora et al., 2020); La nanosílice tiene un efecto en la mejora de las propiedades de transporte de los concretos al refinar la estructura de poros en el rango capilar, originando así una matriz de cemento mucho más impermeable. (Sikora et al., 2020).

\subsection{Nanosílice}

La Nanosílice es un aditivo en estado líquido que procede de las partículas micro finas de dióxido de silicio amorfo $\left(\mathrm{SiO}_{2}\right)$. Las partículas de Nanosílice presentan menor tamaño (vea (Figura 2)), alta pureza y reactividad, una estructura cristalina semejante al humo de sílice, por consiguiente, se espera conseguir superiores resultados con su utilización. Es un nano aditivo en estado líquido, de aspecto turbio y ligeramente viscoso, compuesto a base de sílice con partículas de tamaño nanométrico. Es un gran reductor de agua, de alta actividad y puzolanidad. (Ficha Técnica del aditivo Gaia nanosílice. Cognoscible Tecnologies, s.f.).

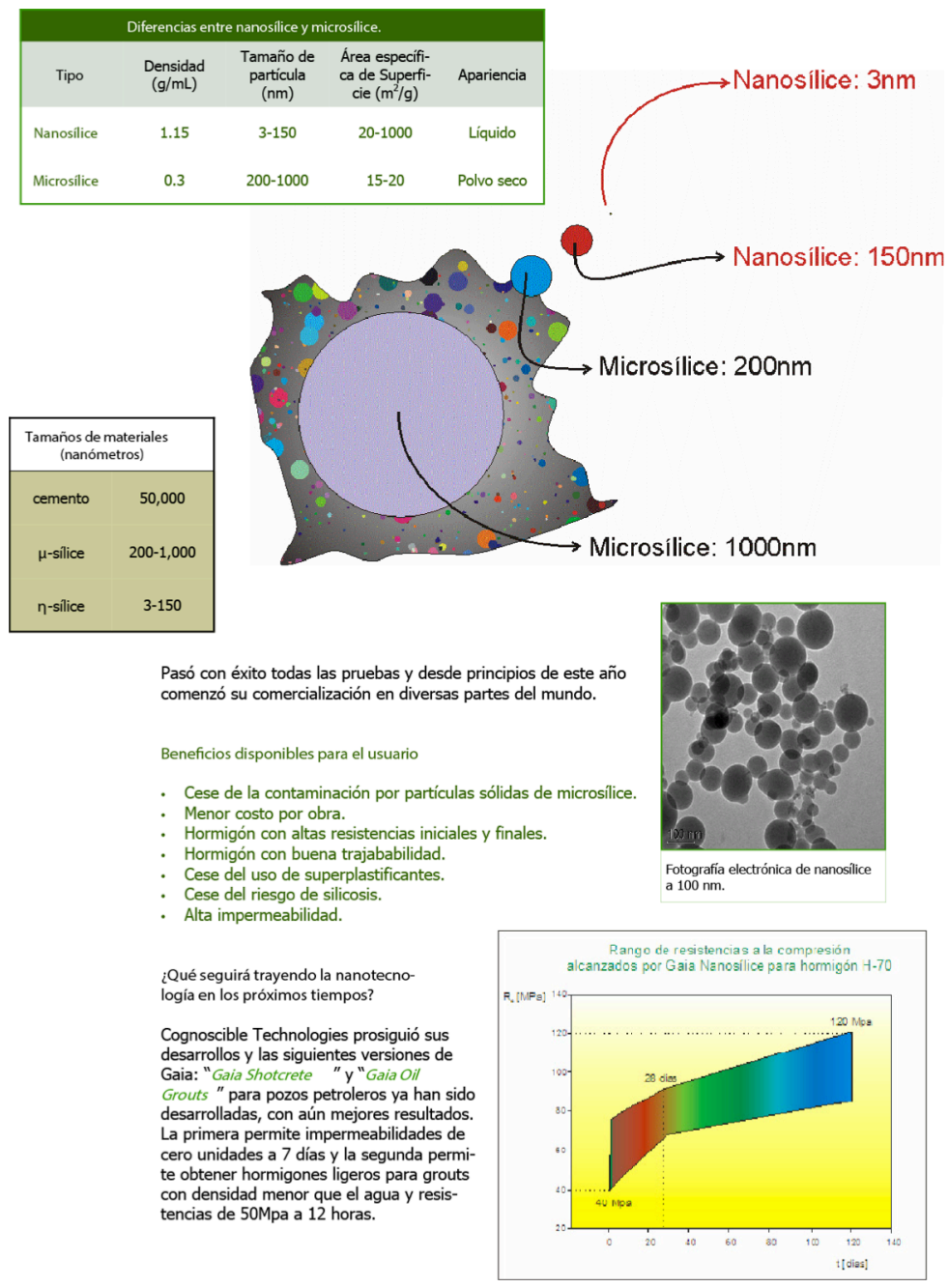

Figura 2. Nanosílice

Fuente: Construcción y tecnología en concreto

Según los estudios, teóricamente las nanopartículas de sílice coloidal reaccionan con los iones de calcio creados durante los periodos de hidratación del cemento (1), conformando un gel de silicato de calcio (C-S-H) (2), (Mehta y Monteiro, 2001). Con distintas palabras, el mecanismo químico es semejante a la reacción puzolánica, sin embargo, con un elemento de alta pureza con una superficie específica muy alta (alrededor de $50000 \mathrm{~m}^{2} / \mathrm{kg}$ ) y 
dinámica como se muestran en las siguientes expresiones (Ecuación 1) y (Ecuación 2), (Dobson y Goberdhan 1988); (Brough y Dobson ,1994).

$$
\begin{aligned}
& \mathrm{C} 3 \mathrm{~S}+\mathrm{H} 2 \mathrm{O}=\mathrm{C}-\mathrm{S}-\mathrm{H} \text { gel }+\mathrm{Ca}(\mathrm{OH}) 2 \\
& \mathrm{SiO} 2(\mathrm{nSA})+\mathrm{Ca}(\mathrm{OH}) 2=\mathrm{C}-\mathrm{S}-\mathrm{H} \text { gel }
\end{aligned}
$$

\section{Programa Experimental}

La presente investigación se desarrollará haciendo uso del estudio experimental. En el estudio experimental, se evalúa el comportamiento a compresión del concreto, utilizando agregado de la cantera de Añashuayco y la utilización de la adición de Nanosilice.

Finalmente, la evaluación y el contraste de resultados experimentales permitirán entender el fenómeno estudiado, y por ende la generación de sugerencias y recomendaciones en el diseño de concretos en base a resultados. Estos propondrán alcanzar concretos capaces de soportar esfuerzos en sus componentes sin que se degrade su resistencia frente a sismos.

\subsection{Cemento puzolánico IP}

Es un cemento fabricado a base de Clinker, con puzolana y yeso; resistente al ataque de sulfatos; el hidróxido de calcio, liberado en la hidratación del cemento, reacciona con los sulfatos.

El cemento portland puzolánico IP produce más cantidad de silicatos, producidos en la hidratación del cemento, disminuyendo la porosidad capilar, así también el concreto se hace más impermeable y protege al acero de la corrosión.

Disminuye la reacción nociva álcali-agregado; menor calor de hidratación; la reacción entre el hidróxido de calcio, liberado en la hidratación del cemento, con el aluminato tricálcico (C3A) presente en el cemento, genera gran calor de hidratación, evitando contracciones y fisuramiento que afecten la calidad del concreto, principalmente en obras de gran volumen.

\subsection{Agregados}

\subsubsection{Caracterización geológica de la quebrada de Añashuayco}

Añashuayco es el nombre con el cual se la denomina a la quebrada que está localizado en la parte noroeste de la ciudad de Arequipa (Perú), Tiene una extensión de varios $\mathrm{km}^{2}$ y ocupa parte del glacis constituida por ignimbritas y depósitos aluvionicos. En el sector de cono norte existen excelentes afloramientos, bien expuestos por las quebradas del Chachani que bajan de las vertientes andinas.

La geomorfología del área presenta una quebrada transversal volcánica proveniente del Chachani y culmina en el margen derecho del rio Chili cerca al batolito de la Caldera en el distrito de Uchumayo.

\subsubsection{Ubicación}

El área de estudio se localiza en las siguientes coordenadas UTM: 8192365 m Norte y 223806 m Este con una altitud de 2545 m.s.n.m., en el asentamiento humano Villa Salvador; y; 8182237 m Norte y 214495 m Este, con una altitud de 1969 m.s.n.m. en la quebrada de Añashuayco, comprende de los distritos de Cerro Colorado y Uchumayo, provincia y departamento de Arequipa.

\subsubsection{Tipos de Agregados y Características:}

Se estudiará el material de la zona de la torrentera y alrededores, de igual manera se ha visto la explotación de los materiales pétreos para la construcción que corresponden a depósitos de abanicos aluviales, los depósitos de conos aluviales corresponden a sedimentos detríticos con arenas y arcillas - limos.

Por consiguiente, las características del agregado fino son la presencia de gran cantidad de limos y arcillas. El agregado grueso es de forma irregular y presenta cristales (que pueden ser de cuarzo) y cierta porosidad.

\subsubsection{Propiedades Físicas del Agregado}

En la (Figura 3) se observa las tres muestras de la granulometría del agregado grueso; en la (Figura 4) las tres muestras del agregado fino. 


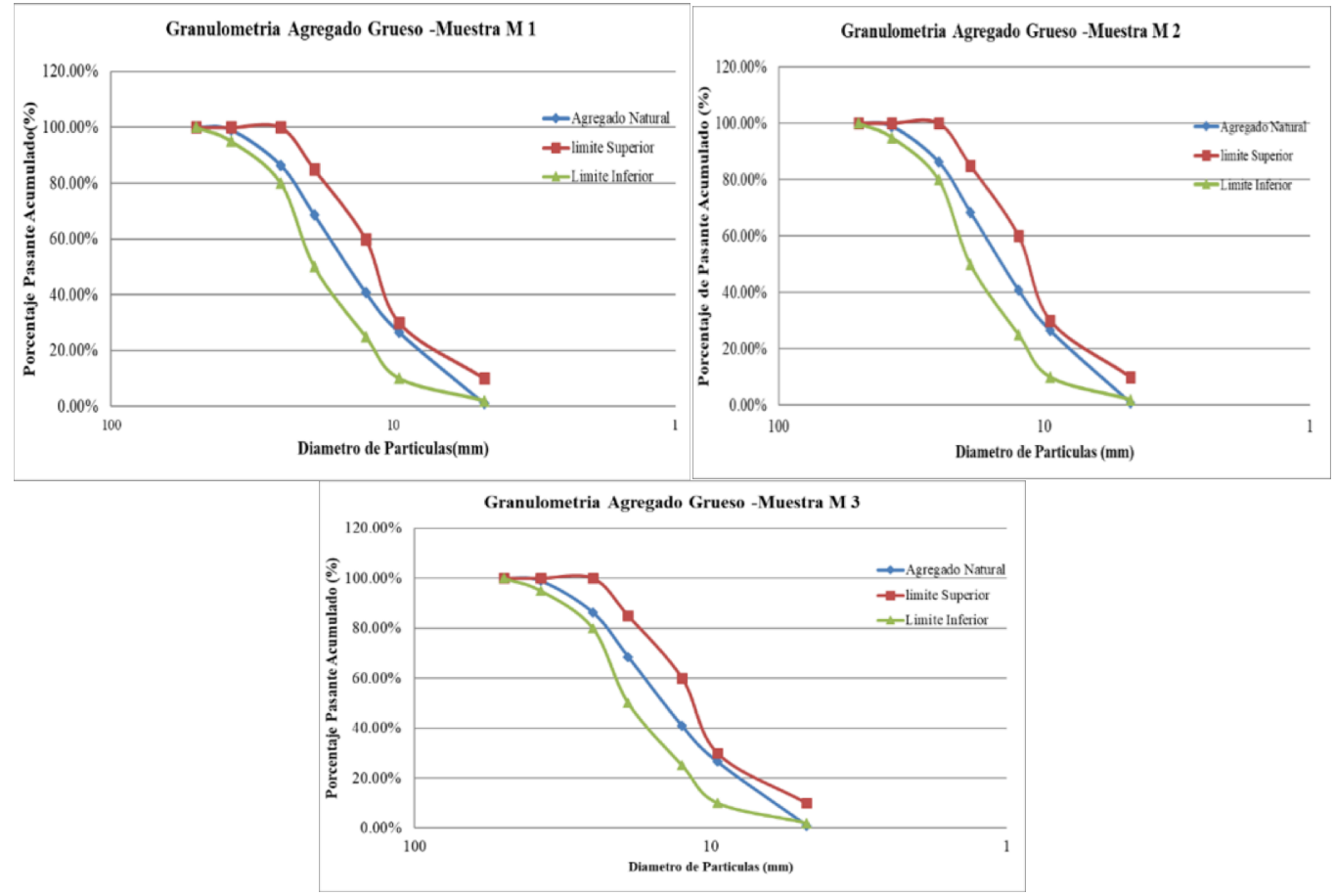

Figura 3. Granulometría del agregado grueso.

Fuente: propia

Resultados: Se realizó la granulometría del agregado grueso. Una vez tamizado el agregado las curvas granulométricas obtenidas de las tres muestras ensayadas se encuentran dentro del uso granulométrico indicado en la NTP 400.037.
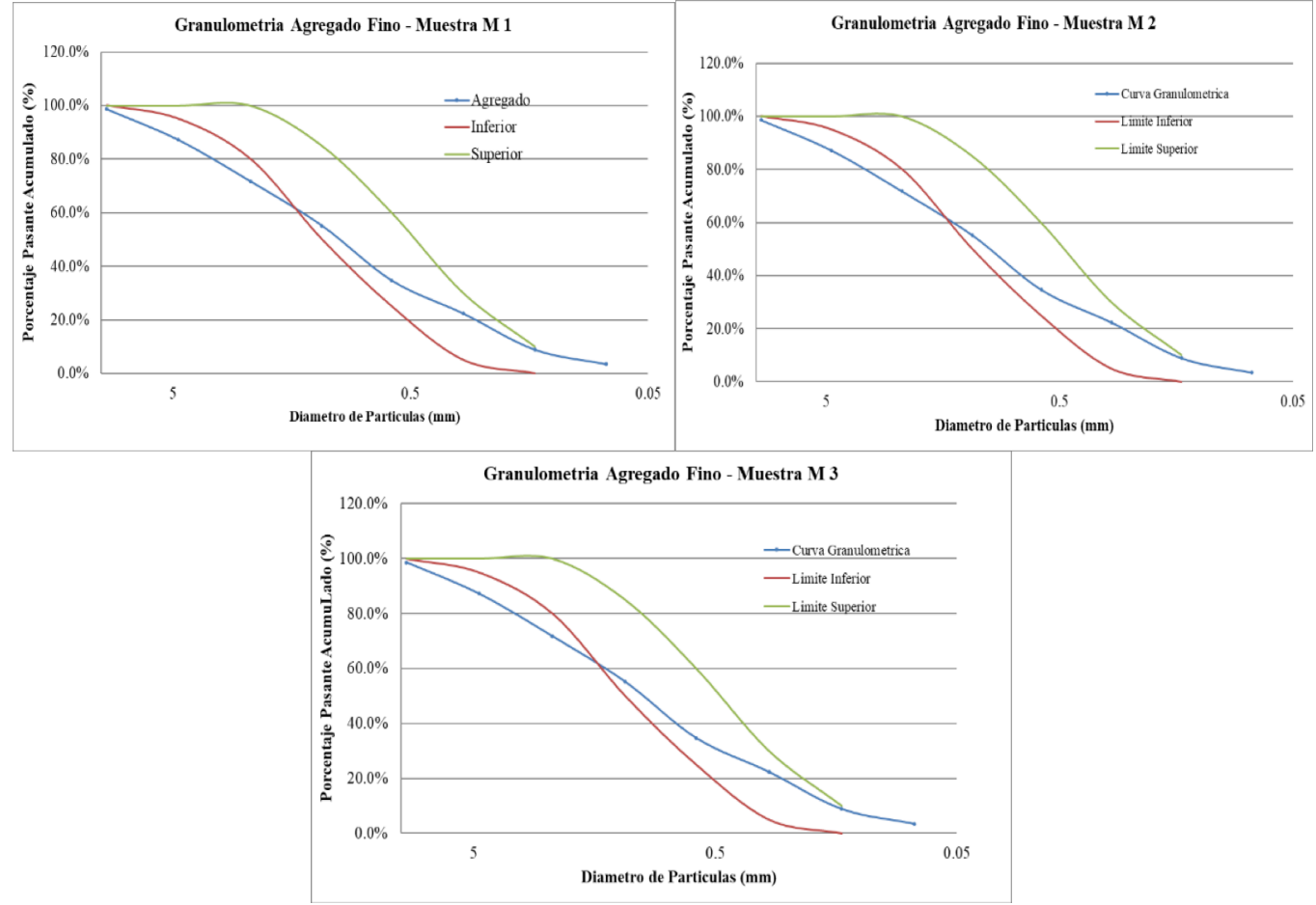

Figura 4. Granulometría del agregado fino Source: Own 
Resultados: Parte de la gráfica granulométrica del agregado fino, se encuentra debajo del límite inferior de la curva establecida en la Norma Técnica Peruana, (NTP 400.037), por lo tanto, es un material no recomendable para el diseño de mezcla; pero mediante un proceso de mejoramiento se puede optimizar para su uso.

Las Propiedades del cemento, agua y agregados utilizados para el diseño de mezclas de concreto se muestra en la (Tabla 1).

Tabla 1. Propiedades del cemento, agua y agregados

\begin{tabular}{|c|c|c|}
\hline \multicolumn{3}{|c|}{ Cemento } \\
\hline Tipo & \multicolumn{2}{|c|}{ Yura IP } \\
\hline Procedencia & \multicolumn{2}{|c|}{ Arequipa-Cono Norte } \\
\hline Peso específico (kg/cm3) & \multicolumn{2}{|c|}{2810} \\
\hline \multicolumn{3}{|c|}{ Agua Potable } \\
\hline Peso específico $(\mathrm{kg} / \mathrm{cm} 3)$ & \multicolumn{2}{|c|}{1000} \\
\hline \multicolumn{3}{|c|}{ Agregados } \\
\hline PROPIEDADES & PROPIEDADES & PROPIEDADES \\
\hline Cantera & Añashuayco & Añashuayco \\
\hline Tamaño Nominal Máximo & \multicolumn{2}{|r|}{$1^{\prime \prime}$} \\
\hline Contenido de Humedad & $0,25 \%$ & $0.09 \%$ \\
\hline Absorción & $0,80 \%$ & $3,21 \%$ \\
\hline Módulo de fineza & 2,54 & 7,16 \\
\hline P.E. de masa $(\mathrm{kg} / \mathrm{cm} 3)$ & 2627 & 2424 \\
\hline P.U. varillado $(\mathrm{kg} / \mathrm{cm} 3)$ & 1677 & 1402 \\
\hline P.U. Suelto seco $(\mathrm{kg} / \mathrm{cm} 3)$ & 1453,7 & 1295,2 \\
\hline
\end{tabular}

\subsection{Agua}

El agua procede de la red pública de agua potable de Arequipa.

\subsection{Adición de Gaia Nanosílice}

Es una productora de nanocemento. La sílice produce partículas de C-S-H, que es el "pegamento" del hormigón y lo que mantiene cohesionado a todas sus partículas de CSH con un tamaño de entre $5 \mathrm{~mm}$ y $250 \mathrm{~mm}$ (Ficha Técnica del aditivo Gaia nanosílice. Cognoscible Tecnologies, s.f.).

\section{Diseño de mezclas}

Para el diseño del concreto patrón (concreto convencional) y para el diseño de los concretos no convencionales (con la adición de Nanosílice) se aplicó la siguiente secuencia; la cantidad de agua se mantiene según el diseño, también se mantuvo la proporción de agregados y cemento que se utilizó; el único porcentaje que cambia es la Nanosílice en porcentajes de $0.6 \%, 0.8 \%, 1.0 \%$ y $1.4 \%$ para las respectivas resistencias. En la (Tabla 2) se puede ver la representación de estos datos.

El agua se añadió continuamente a la mezcla, hasta que obtengamos una pasta bien mezclada, después las probetas se colocaron en una poza de curado en las mismas condiciones de humedad y temperatura. 
Table 2. Resultados de las proporciones de los materiales

\begin{tabular}{|c|c|c|c|c|c|}
\hline Material & $\mathbf{1 7 5} \mathbf{~ k g} / \mathbf{c m} \mathbf{2}$ & $\mathbf{2 1 0} \mathbf{~ k g} / \mathbf{c m} \mathbf{2}$ & $\mathbf{2 8 0} \mathbf{~ k g} / \mathbf{c m} 2$ & $\mathbf{3 5 0} \mathbf{~ k g} / \mathbf{c m} 2$ & Unidad \\
\hline Cemento & 326,433 & 367,12 & 439,914 & 517,68 & $\mathrm{~kg} / \mathrm{cm} 3$ \\
\hline Agregado fino & 751,004 & 713,798 & 645,478 & 572,495 & $\mathrm{~kg} / \mathrm{cm} 3$ \\
\hline Agregado grueso & 906,198 & 906,578 & 906,578 & 906,578 & $\mathrm{~kg} / \mathrm{cm} 3$ \\
\hline Agua efectiva & 238,638 & 237,121 & 236,843 & 236,547 & $\mathrm{~kg} / \mathrm{cm} 3$ \\
\hline Nanosílice 0,6\% & 1,908 & 1,999 & 2,570 & 3,024 & $\mathrm{~kg} / \mathrm{cm} 3$ \\
\hline Nanosílice 0,8\% & 2,542 & 2,665 & 3,426 & 4,032 & $\mathrm{~kg} / \mathrm{cm} 3$ \\
\hline Nanosílice 1\% & 3,332 & 3,574 & 4,283 & 5,041 & $\mathrm{~kg} / \mathrm{cm} 3$ \\
\hline Nanosílice 1,4\% & 4,664 & 5,005 & 5,997 & 7,056 & $\mathrm{~kg} / \mathrm{cm} 3$ \\
\hline
\end{tabular}

\section{Resulltados y discusión}

\subsection{Slump}

En la (Tabla 3) se muestran los resultados del Slump para 4 dosificaciones establecidas de resistencia a compresión simple de concreto estructural $\left(175,210,280\right.$ y $\left.350 \mathrm{Kg} / \mathrm{cm}^{2}\right)$ y diferentes porcentajes de nanosílice en peso de cemento). En la (Figura 5) se observan los resultados de Slump para la situación establecida. Del análisis de resultados de la tabla, se observó que para incrementos en la dosis de aditivo Nanosílice $(0,6 \% ; 0,8 \% ; 1 \%$ y $1,4 \%$ respecto al peso del cemento), el asentamiento del concreto se encuentra en el rango de 2 a 4 pulgadas. Además, se observa que el valor mínimo de Slump para cada dosificación de concreto es: para $175 \mathrm{Kg} / \mathrm{cm}^{2}$ es $23 / 4$ pulgadas, para $210 \mathrm{Kg} / \mathrm{cm}^{2}$ es 3 3/4 pulgadas, para $280 \mathrm{Kg} / \mathrm{cm}^{2}$ es 2 pulgadas y para $350 \mathrm{Kg} / \mathrm{cm}^{2}$ es 2 1/2 pulgadas.

Tabla 3. Resultados del Slump -Método de Aci (Fuente Propia)

\begin{tabular}{|c|c|c|c|}
\hline$\underset{\text { ID }}{\text { MEZCLA }}$ & $\begin{array}{c}\text { CONCRETO } \\
\text { ESTRUCTURAL F'c } \\
\left(\mathrm{Kg} / \mathrm{cm}^{2}\right) \\
\end{array}$ & $\begin{array}{c}\text { DOSIFICACION DE ADITIVO } \\
\text { NANOSILICE ( } \% \text { del peso de } \\
\text { cemento) }\end{array}$ & $\begin{array}{c}\text { SLUMP } \\
\text { MÁXIMO(pulgadas) }\end{array}$ \\
\hline 1 & \multirow{5}{*}{175} & 0 & 4 \\
\hline 2 & & 0.60 & $31 / 2$ \\
\hline 3 & & 0.80 & $23 / 4$ \\
\hline 4 & & 1.00 & $\begin{array}{ll}3 & 1 / 4 \\
\end{array}$ \\
\hline 5 & & 1.40 & $4 \quad 1 / 8$ \\
\hline 1 & \multirow{5}{*}{210} & 0 & 4 \\
\hline 2 & & 0.60 & 4 \\
\hline 3 & & 0.80 & $4 \quad 1 / 2$ \\
\hline 4 & & 1.00 & 4 \\
\hline 5 & & 1.40 & $33 / 4$ \\
\hline 1 & \multirow{5}{*}{280} & 0 & $21 / 4$ \\
\hline 2 & & 0.60 & 3 \\
\hline 3 & & 0.80 & 3 \\
\hline 4 & & 1.00 & 2 \\
\hline 5 & & 1.40 & $21 / 2$ \\
\hline 1 & \multirow{5}{*}{350} & 0 & $23 / 4$ \\
\hline 2 & & 0.60 & $21 / 4$ \\
\hline 3 & & 0.80 & 3 \\
\hline 4 & & 1.00 & $21 / 2$ \\
\hline 5 & & 1.40 & $4 \quad 1 / 2$ \\
\hline
\end{tabular}




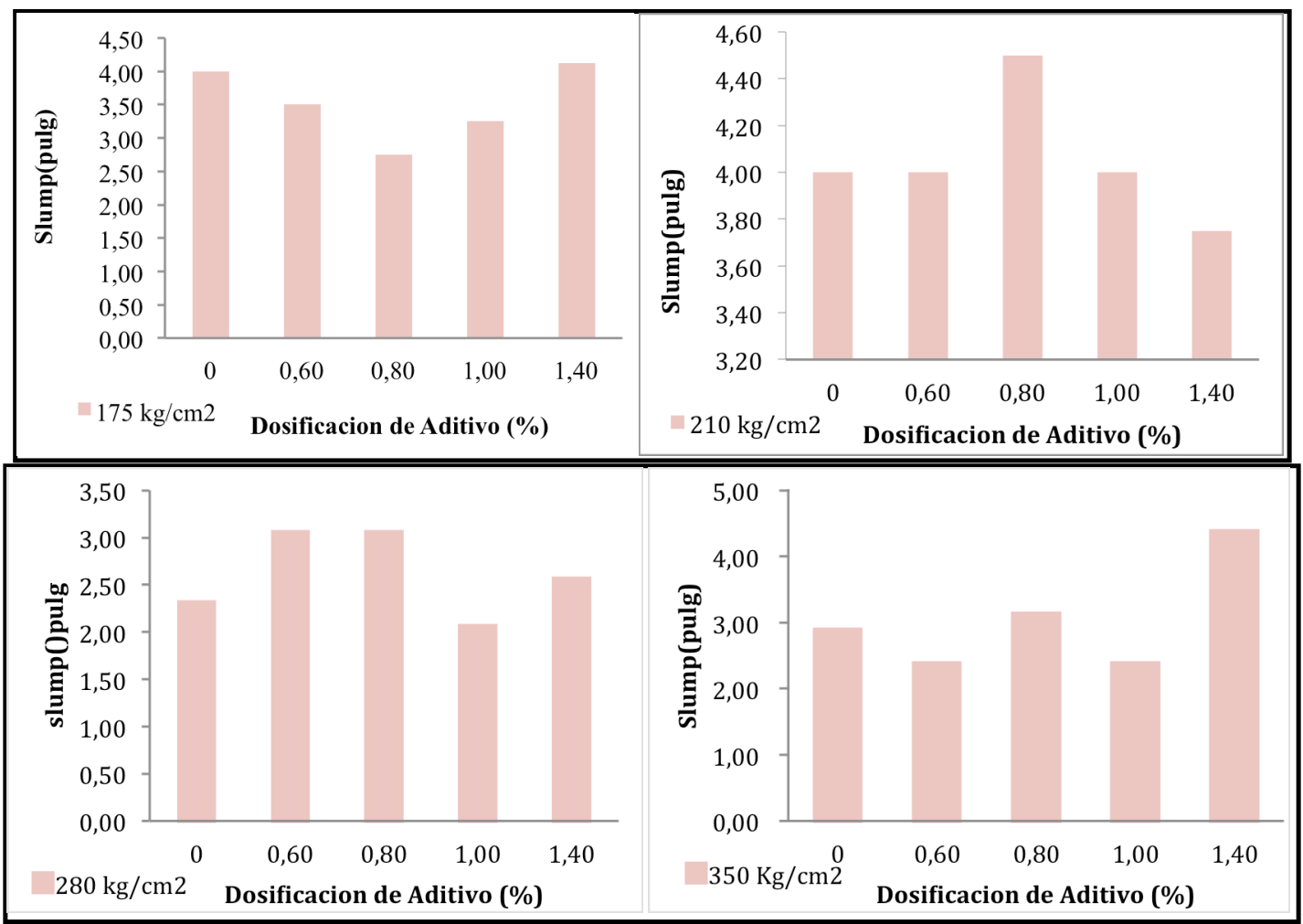

Figura 5. Ensayo de Asentamiento para una resistencia de 175,210,280,350 kg/cm2.

Fuente: propia

\subsection{Contenido de aire}

Según los resultados obtenidos de contenido de aire, son menores o iguales 3,5\% para los distintos diseños de concreto con y sin Nanosílice ver (Figura 6) y (Tabla 4).

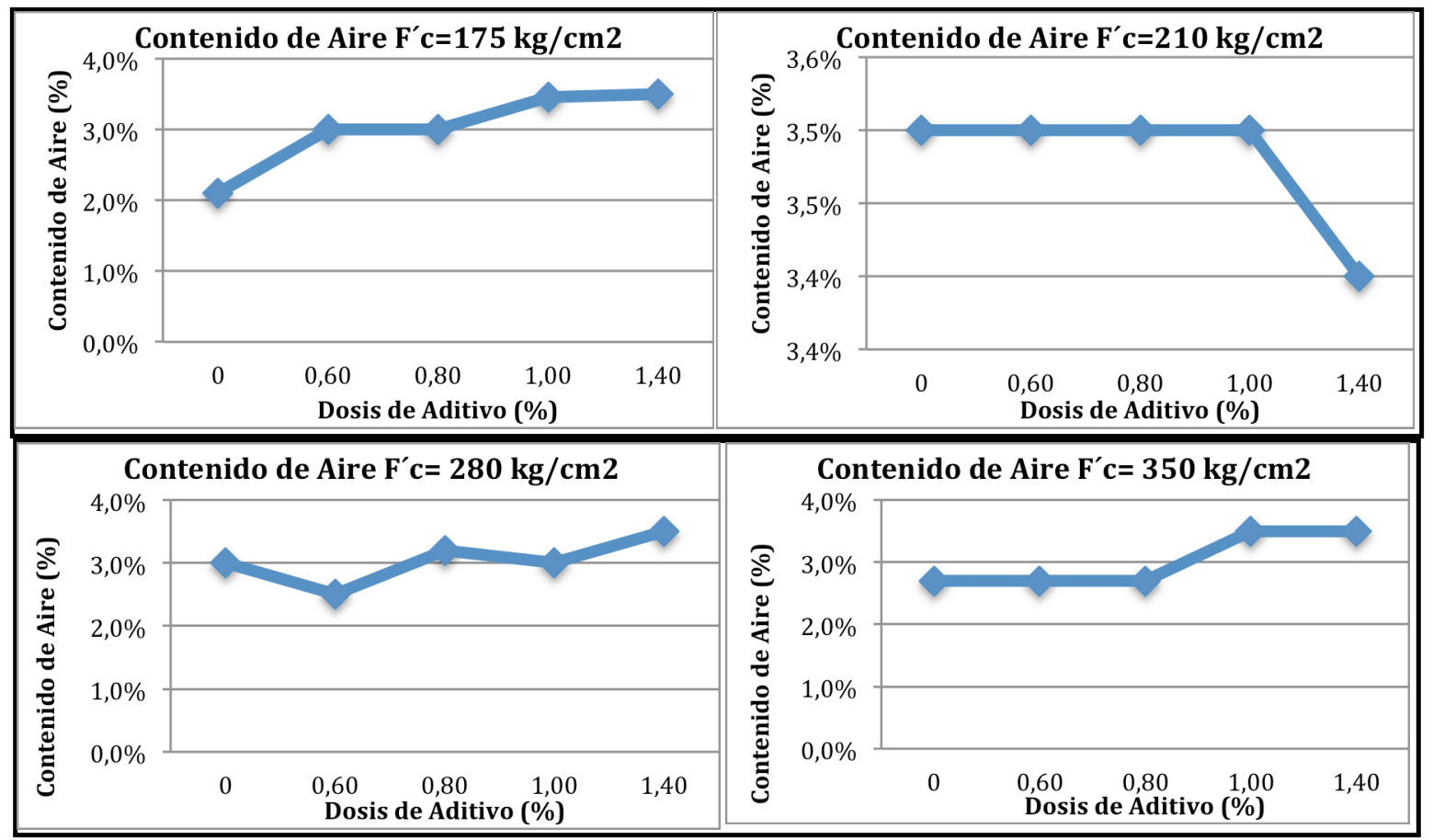


Figura 6. Ensayo de Contenido de Aire para un $\mathrm{F}^{\prime} \mathrm{C}=175,210,280,350 \mathrm{~kg} / \mathrm{cm} 2$.Fuente: propia

Tabla 4. Resultados del Contenido de Aire del Concreto Fresco - Método de Aci

\begin{tabular}{|c|c|c|c|c|}
\hline $\begin{array}{c}\text { ID } \\
\text { MEZCLA }\end{array}$ & 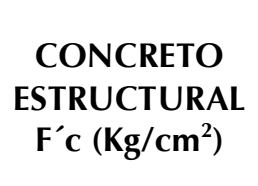 & $\begin{array}{l}\text { DOSIFICACIÓN DE } \\
\text { ADITIVO } \\
\text { NANOSILICE (\% del } \\
\text { peso de cemento) }\end{array}$ & $\begin{array}{l}\text { CONTENIDO } \\
\text { DE AIRE }(\%)\end{array}$ & $\begin{array}{c}\% \text { DE VARIACION } \\
\text { CON RESPECTO } \\
\text { AL CONCRETO SIN } \\
\text { ADITIVO }\end{array}$ \\
\hline 1 & \multirow{5}{*}{175} & 0 & $2.1 \%$ & $100 \%$ \\
\hline 2 & & 0.60 & $3.0 \%$ & 142.86 \\
\hline 3 & & 0.80 & $3.0 \%$ & 142.86 \\
\hline 4 & & 1.00 & $3.5 \%$ & 164.29 \\
\hline 5 & & 1.40 & $3.4 \%$ & 166.67 \\
\hline 1 & \multirow{5}{*}{210} & 0 & $3.5 \%$ & $100 \%$ \\
\hline 2 & & 0.60 & $2.2 \%$ & 62.86 \\
\hline 3 & & 0.80 & $3.3 \%$ & 100.00 \\
\hline 4 & & 1.00 & $2.2 \%$ & 62.86 \\
\hline 5 & & 1.40 & $3.4 \%$ & 97.14 \\
\hline 1 & \multirow{5}{*}{280} & 0 & $3.0 \%$ & $100 \%$ \\
\hline 2 & & 0.60 & $2.5 \%$ & 83.33 \\
\hline 3 & & 0.80 & $3.2 \%$ & 106.67 \\
\hline 4 & & 1.00 & $3.0 \%$ & 100.00 \\
\hline 5 & & 1.40 & $3.4 \%$ & 116.67 \\
\hline 1 & \multirow{5}{*}{350} & 0 & $2.7 \%$ & $100 \%$ \\
\hline 2 & & 0.60 & $2.7 \%$ & 100.00 \\
\hline 3 & & 0.80 & $2.7 \%$ & 100.00 \\
\hline 4 & & 1.00 & $3.5 \%$ & 129.63 \\
\hline 5 & & 1.40 & $3.4 \%$ & 129.63 \\
\hline
\end{tabular}

\subsection{Peso unitario}

El Peso Unitario obtenido para los diseños están en el rango entre 2201,503 y 2283,655 kg/m $\mathrm{m}^{3}$. Sin embargo, los valores obtenidos a partir de los ensayos corresponden a un concreto normal, para todos los diseños con y sin adición de Nanosílice. Ver (Tabla 5) y (Figura 7). 
Tabla 5. Resultados del Ensayo de Peso Unitario de Concreto

\begin{tabular}{|c|c|c|c|c|c|c|c|}
\hline$\underset{\text { MEZCLA }}{\text { ID }}$ & 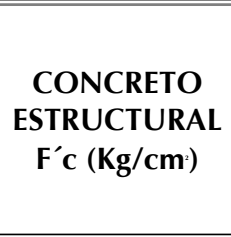 & $\begin{array}{c}\text { DOSIFICACI } \\
\text { ON DE } \\
\text { ADITIVO } \\
\text { NANOSILICE } \\
\text { (\% del peso } \\
\text { de cemento) } \\
\end{array}$ & $\begin{array}{l}\text { PESO DEL } \\
\text { MOLDE } \\
(\text { kgf })\end{array}$ & $\begin{array}{l}\text { PESO DEL } \\
\text { MOLDE } \\
+ \text { CONCRE } \\
\text { TO(kgf) }\end{array}$ & $\begin{array}{l}\text { PESO DEL } \\
\text { CONCRET } \\
\text { O(kgf) }\end{array}$ & $\begin{array}{l}\text { VOL. } \\
\text { MOLDE } \\
(\mathbf{c m})\end{array}$ & $\begin{array}{c}\text { PESO } \\
\text { UNITARIO(kgf/ } \\
\text { m })\end{array}$ \\
\hline 1 & \multirow{5}{*}{175} & 0 & 4.3 & 11.450 & 7.150 & 3152.84 & 2267.797 \\
\hline 2 & & 0.60 & 4.3 & 11.340 & 7.040 & 3152.84 & 2232.907 \\
\hline 3 & & 0.80 & 4.3 & 11.340 & 7.040 & 3152.84 & 2232.907 \\
\hline 4 & & 1.00 & 4.3 & 11.200 & 6.900 & 3152.84 & 2201.503 \\
\hline 5 & & 1.40 & 4.3 & 11.445 & 7.145 & 3152.84 & 2266.211 \\
\hline 1 & \multirow{5}{*}{210} & 0 & 4.3 & 11.210 & 6.910 & 3152.84 & 2211.675 \\
\hline 2 & & 0.60 & 4.3 & 11.250 & 6.950 & 3152.84 & 2204.362 \\
\hline 3 & & 0.80 & 4.3 & 11.200 & 6.900 & 3152.84 & 2201.503 \\
\hline 4 & & 1.00 & 4.3 & 11.300 & 7.000 & 3152.84 & 2220.220 \\
\hline 5 & & 1.40 & 4.3 & 11.400 & 7.100 & 3152.84 & 2251.938 \\
\hline 1 & \multirow{5}{*}{280} & 0 & 4.3 & 11.300 & 7.000 & 3152.84 & 2220.220 \\
\hline 2 & & 0.60 & 4.3 & 11.300 & 7.000 & 3152.84 & 2220.220 \\
\hline 3 & & 0.80 & 4.3 & 11.340 & 7.040 & 3152.84 & 2232.907 \\
\hline 4 & & 1.00 & 4.3 & 11.500 & 7.200 & 3152.84 & 2283.655 \\
\hline 5 & & 1.40 & 4.3 & 11.300 & 7.000 & 3152.84 & 2220.220 \\
\hline 1 & \multirow{5}{*}{350} & 0 & 4.3 & 11.250 & 6.950 & 3152.84 & 2204.362 \\
\hline 2 & & 0.60 & 4.3 & 11.350 & 7.050 & 3152.84 & 2236.079 \\
\hline 3 & & 0.80 & 4.3 & 11.355 & 7.055 & 3152.84 & 2237.665 \\
\hline 4 & & 1.00 & 4.3 & 11.200 & 6.900 & 3152.84 & 2201.503 \\
\hline 5 & & 1.40 & 4.3 & 11.350 & 7.050 & 3152.84 & 2236.079 \\
\hline
\end{tabular}
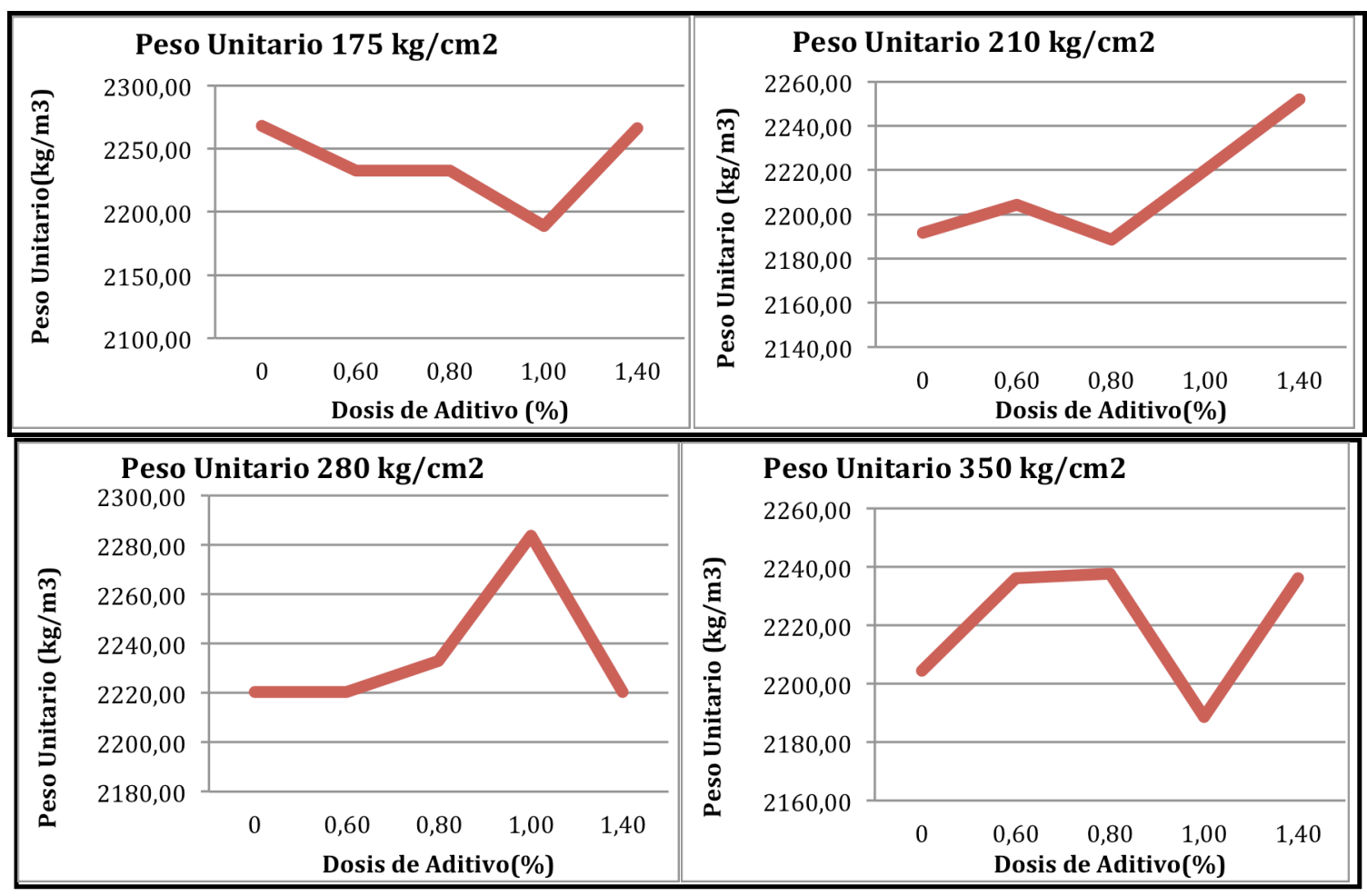

Figura 7. Ensayo de Peso Unitario para una resistencia de 175,210,280,350 kg/ $\mathrm{cm}^{2}$. 


\subsection{Resistencia a la Compresión}

Se puede observar en la (Figura 8) el crecimiento de resistencia a compresión que le otorga el aditivo con Nanosílice, en consecuencia, como ya se mencionó anteriormente Mehta y Monteiro determinaron que la presencia de Sílice (Nanosílice) reduce el espesor de la zona de transición entre pasta y el agregado dando lugar a la reducción de exudación. Esto se debe a 2 motivos importantes, el primero es el refinamiento de los poros asociados principalmente al efecto nano-relleno donde llena parcialmente los huecos grandes y los poros capilares para refinar la estructura de poro de la pasta de cemento. El segundo motivo es la reacción puzolanica de la NS puede consumir portadita, no solo reduce el hidróxido de calcio de la pasta de cemento, sino que también causa los productos de hidratación calcio de la pasta para volverse más homogéneo (C-S-H).

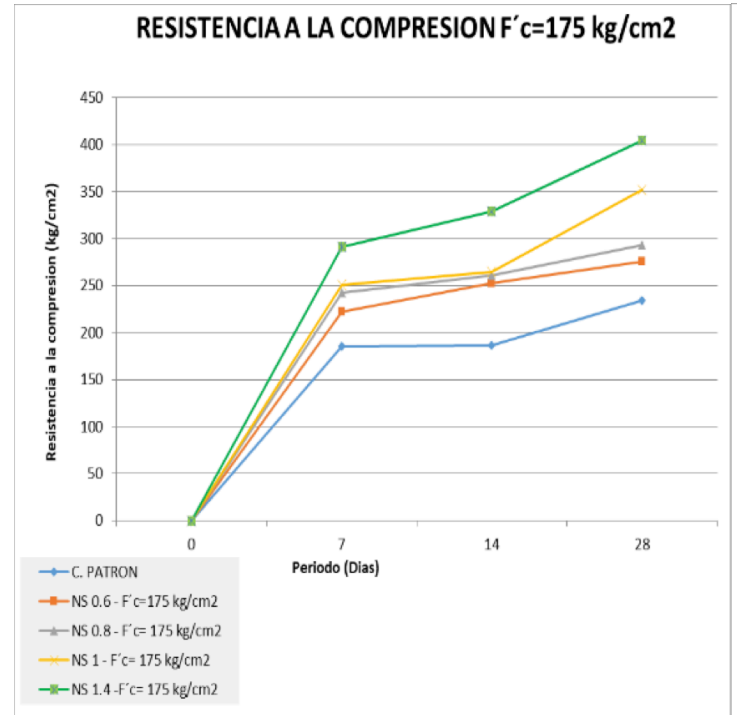

RESISTENCIAA LA COMPRESION F'c $=210 \mathrm{~kg} / \mathrm{cm} 2$

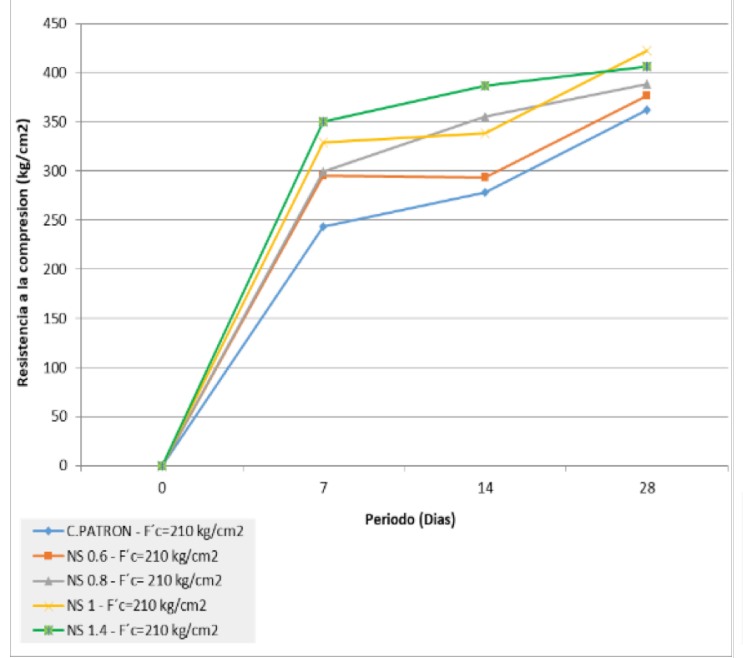

RESISTENCIAA LA COMPRESIONF' $\mathrm{c}=280 \mathrm{~kg} / \mathrm{cm} 2$

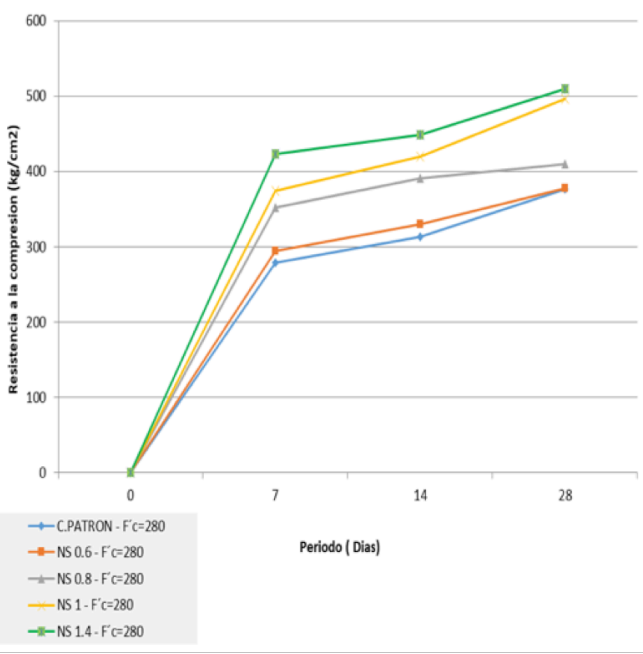

RESISTENCIAA LA COMPRESIONF'c $=350 \mathrm{KG} / \mathrm{CM} 2$

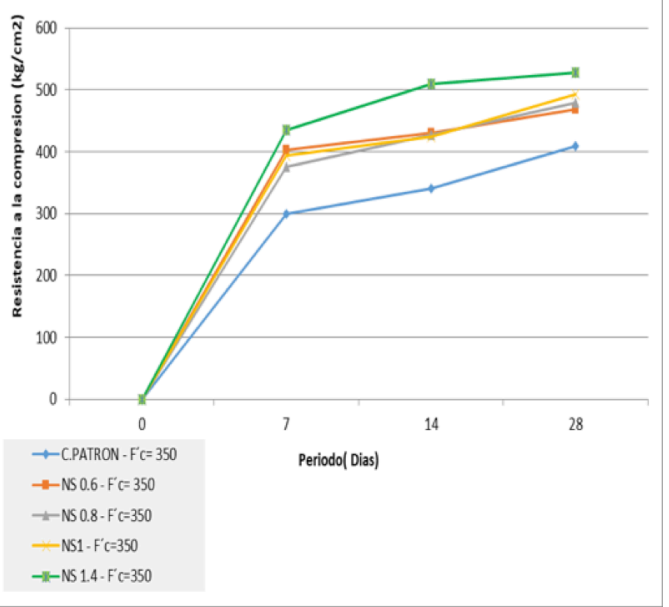

Figura 8. Ensayo de resistencia a la compresión para una resistencia de $175 \mathrm{~kg} / \mathrm{cm}^{2}$. Fuente: propia

Podemos notar en la (Figura 8) el incremento de resistencia de los concretos con Nanosílice; por ejemplo, las probetas con NS 0.6, NS 0.8, NS 1 y NS 1.4 tenían una resistencia a la compresión de $175 \mathrm{~kg} / \mathrm{cm}^{2}$ a los 7 días, lo que mostraba relaciones de incremento de $20.24 \%, 31.19 \%, 35.63 \%$ y $57.29 \%$ en comparación con los grupos de referencia (concreto - patrón). Mientras tanto, NS también contribuyo a la resistencia a la compresión a los 28 días, que tuvo índices de incremento del $17.33 \%, 24.89 \%, 49.84 \%$ y $72.38 \%$ para NS 0.6 , NS 0.8 , NS 1 y NS 1.4 
en comparación de las probetas con concreto patrón. En la (Figura 9) se puede observar la rotura de la probeta de concreto.

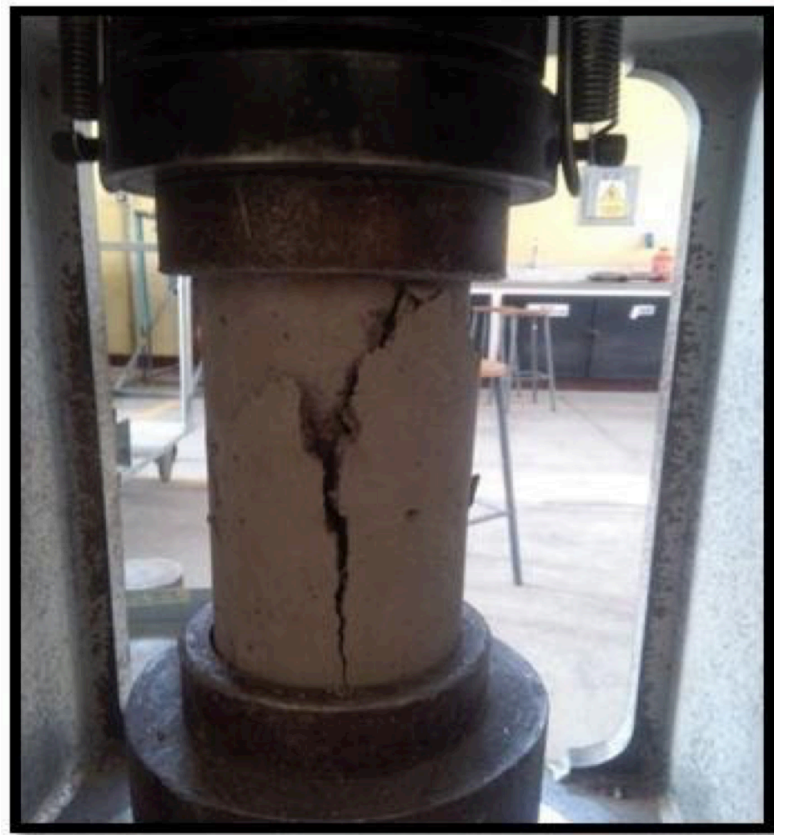

Figure 9. Ensayo de resistencia a la compresión.

Fuente: propia

\subsection{Permeabilidad (D.I.N. 1045, D.I.N. 1048)}

Se observa en la (Tabla 6) y (Figura 10), que un concreto normal sin adición de Nanosílice tiene mayor penetración que los concretos con adición de Nanosílice. También podemos ver en la (Tabla 6) que la penetración es muy alta en un concretó patrón, a diferencia de un concreto con aditivo donde se observa que disminuye un $50 \%$ en penetración. El ensayo por permeabilidad se puede ver en la (Figura 11).

Tabla 6. Resultados de los ensayos de penetración para resistencias de 210 kg/ $\mathrm{cm}^{2}$

\begin{tabular}{|c|c|c|c|c|c|c|}
\hline $\begin{array}{c}\text { RESISTENCIA } \\
\text { DE ESTUDIO } \\
\left(\mathbf{K g} / \mathbf{c m}^{\mathbf{2}}\right)\end{array}$ & $\begin{array}{c}\text { DOSIFICACION } \\
\text { DE ADITIVO } \\
\text { NANOSILICE } \\
\text { \% del peso de } \\
\text { cemento) }\end{array}$ & $\begin{array}{c}\text { EDAD } \\
(\mathbf{d i ́ a s )}\end{array}$ & $\begin{array}{c}\text { Penetració } \\
\mathbf{n} \mathbf{1 - ( \mathbf { m m } )}\end{array}$ & $\begin{array}{c}\text { Penetració } \\
\mathbf{n} \mathbf{2}-\mathbf{( m m})\end{array}$ & $\begin{array}{c}\text { Penetración } \\
\mathbf{3}-\mathbf{( m m})\end{array}$ & $\begin{array}{c}\text { PENETRACION } \\
\text { PROMEDIO(mm) }\end{array}$ \\
\hline 210 & 0,0 & 28 & 24,9 & 25,4 & 25,6 & 25,30 \\
\hline 210 & 0,6 & 28 & 14,2 & 8,9 & 8,2 & 10,43 \\
\hline 210 & 1,0 & 28 & 10,4 & 9,6 & 8,0 & 9,33 \\
\hline 210 & 1,4 & 28 & 7,5 & 15,4 & 8,5 & 10,47 \\
\hline
\end{tabular}




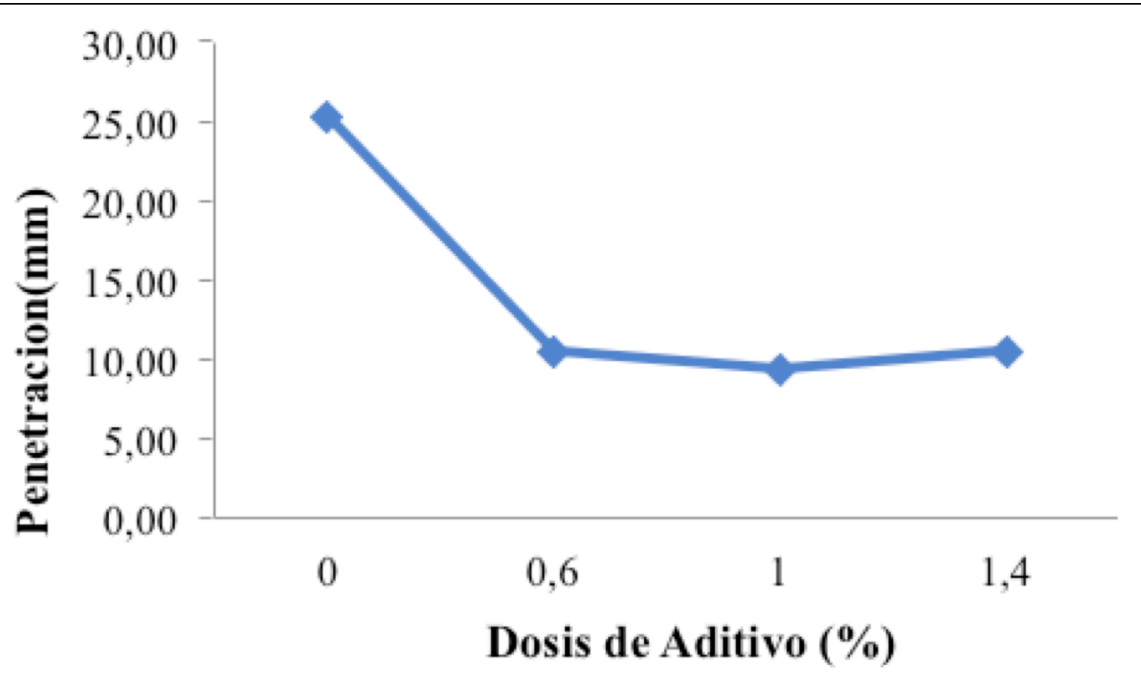

Figura 10. Ensayo de Permeabilidad. Fuente: propia

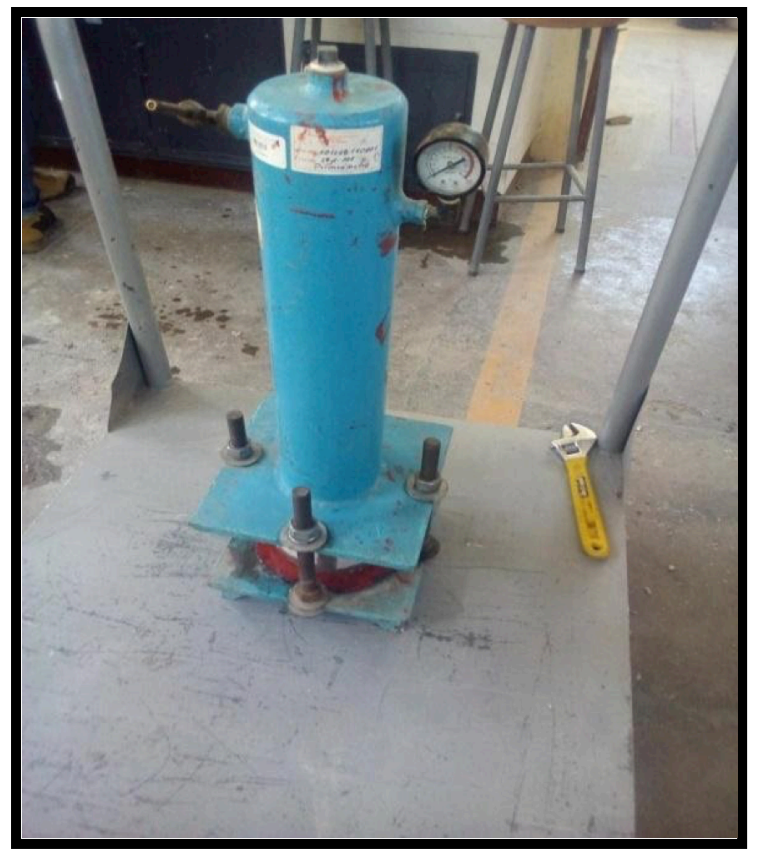

Figura 11. Ensayo de Permeabilidad para una resistencia de $210 \mathrm{~kg} / \mathrm{cm}^{2}$. Fuente: propia 
3.6 Análisis comparativo de costos del concreto con Nanosílice comparado con el concreto sin Nanosílice y concreto con agregado de la poderosa.

La composición del nuevo concreto se diseñó en base al concepto de Mehta y Monteiro; la cantidad de material utilizado definido en costos, se aprecia en la (Tabla 7) y (Figura 12).

Tabla 7. Comparación de costos para resistencias de 175,210,280 y 350 kg/cm2

\begin{tabular}{|c|c|}
\hline Nombre & Costo(s/.) \\
\hline C.Poderosa & 208.761 \\
\hline C.Patron 175 & 172.601 \\
\hline NS $0.6-175$ & 202.195 \\
\hline NS $0.8-175$ & 212.059 \\
\hline NS $1-175$ & 231.687 \\
\hline NS $1.4-175$ & 252.381 \\
\hline Nombre & Costo(s/.) \\
\hline C.Poderosa & 254.43 \\
\hline C.Patron 210 & 191.763 \\
\hline NS $0.6-210$ & 210.992 \\
\hline NS $0.8-210$ & 221.339 \\
\hline NS $1-210$ & 245.914 \\
\hline NS $1.4-210$ & 269.485 \\
\hline Nombre & Costo(s/.) \\
\hline C.Poderosa & 345.52 \\
\hline C.Patron 350 & 265.086 \\
\hline NS $0.6-350$ & 312.049 \\
\hline NS $0.8-350$ & 327.698 \\
\hline NS $1-350$ & 343.362 \\
\hline NS $1.4-350$ & 374.661 \\
\hline Nombre & Costo(s/.) \\
\hline C. Poderosa & 304,590 \\
\hline C. Patron 280 & 227,216 \\
\hline NS $0,6-280$ & 267,123 \\
\hline NS $0,8-280$ & 280,408 \\
\hline NS $1,0-280$ & 293,729 \\
\hline NS 1,4 - 280 & 320,340 \\
\hline
\end{tabular}



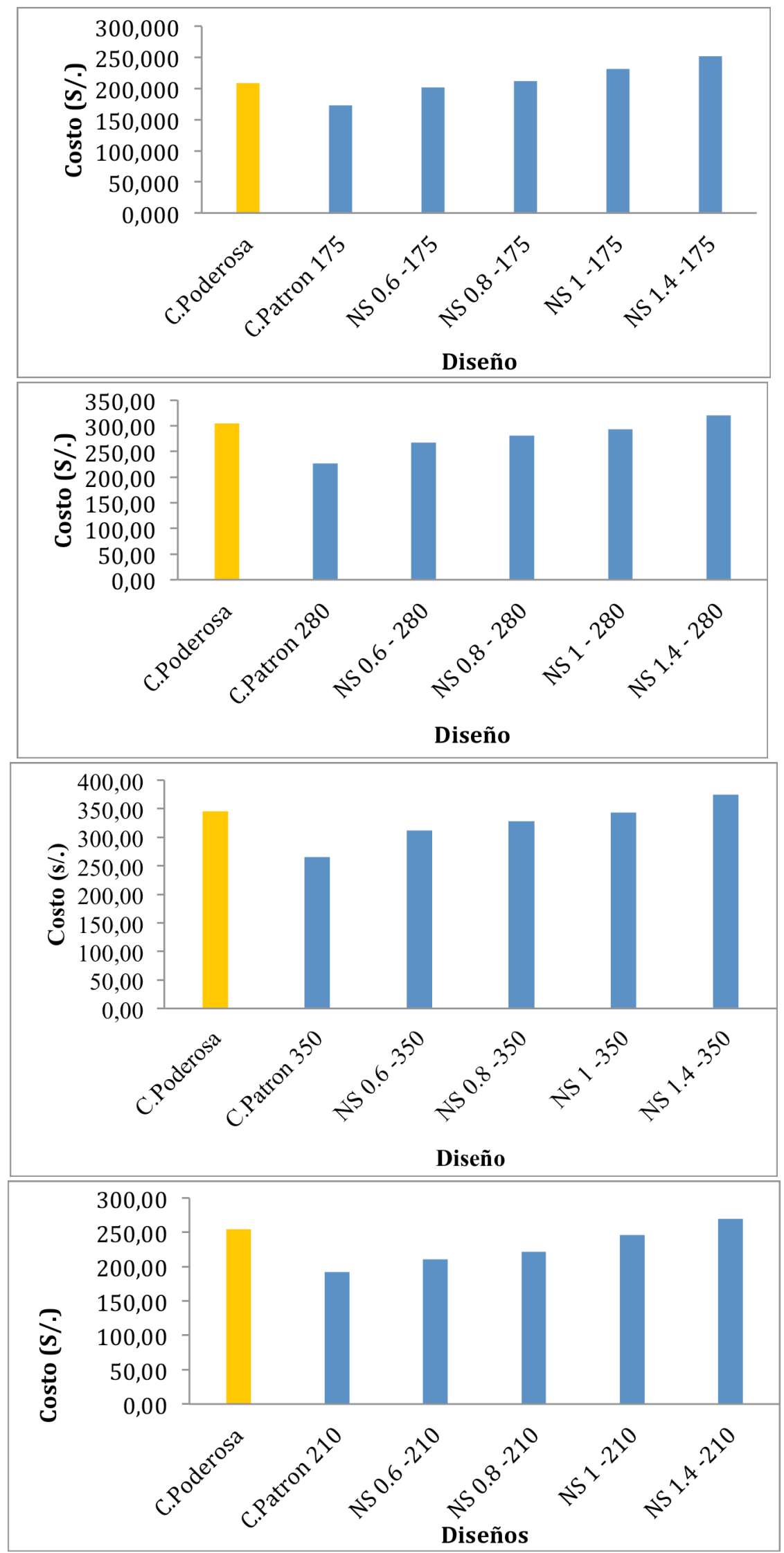

Figure 12. Comparación de costos.

Fuente: propia 
Podemos concluir que los diseños con agregados de Añashuayco y con adición de Nanosílice entre el rango de 0,8 a 1,4 \% alcanzan las resistencias de un diseño con agregado de la poderosa, pero en cuanto a costos los diseños con agregados de Añashuayco son más económicos. Pero tenemos que tener en cuenta que concretos con adiciones de Nanosílice mayores al $1 \%$ el costo es mayor que un concreto que usa agregados de la cantera la poderosa.

\section{Conclusiones}

En el presente estudio se analizó el beneficio de incorporar agregados fino y grueso de la cantera de Añashuayco a una mezcla optima con Nanosílice respecto a concreto patrón obtenidos a 28 días de edad, para desarrollar un tipo de concreto de alto desempeño CAD:

Se concluye que la resistencia a compresión mejoró sustancialmente, gracias a la reacción puzolanica de la Nanosílice con la portlandita de manera rápida producen gel C-S-H, el cual reduce la porosidad de las estructuras. Además, el tamaño nanometrico de las partículas de Nanosílice puede llenar parcialmente los poros capilares para refinar la estructura de poro de la pasta de cemento.

Los agregados de la cantera Añashuayco no son de buena calidad, limitando su resistencia, el uso de adición de Nanosílice para agregados similares, favorecerá la resistencia y optimización del concreto.

Los valores de desviación estándar de los ensayos de compresión varían entre $4,3 \mathrm{~kg} / \mathrm{cm}^{2}$ hasta $21 \mathrm{~kg} / \mathrm{cm}^{2}$, este resultado comparado con la tabla de control de calidad para especímenes aporta una calificación de buenos y excelentes en su mayoría. Se puede establecer la calidad y confiabilidad de los resultados según los procedimientos dados por el comité ACl 214.

La permeabilidad de los concretos con aditivo se reduce progresivamente de acuerdo al porcentaje de aditivo, aportando mayor compacidad al concreto, se observa que disminuye un $50 \%$ en penetración. Hace que el concreto este más compacto, al punto de lograr que los elementos que causan la corrosión no puedan penetrar el hormigón.

Se puede observar que los testigos de concreto para diseños de $280 \mathrm{~kg} / \mathrm{cm}^{2}$ con agregados de la poderosa resultan muy costosos con precio aproximado de S/. 304,59 (sin considerar el flete del agregado). El concreto obtenido tiene un costo aproximado de S/. 267,123 siendo 15\% más económico. Se puede concluir que concretos con agregado de Añashuayco adicionando el porcentaje de Nanosílice alcanza los requerimientos de resistencias para un concreto estructural y además genera un beneficio económico al ser un concreto muy accesible en costos a la población de Cono Norte.

\section{Agradecimientoss}

- Los autores agradecen a la "Universidad Nacional de San Agustín de Arequipa" por la subvención otorgada con contrato $N^{\circ}$ TP-027-2018-UNSA.

- Por la beca otorgada en la consecución de sus estudios de Titulación y por compartir sus laboratorios para concluir este estudio.

- También agradecemos el apoyo brindado para la realización del presente estudio a Unsa Investiga de Arequipa.

\section{Referencias}

American Concrete Institute (2017). ACl Comité ACl-214: Prácticas recomendadas para la evaluación de resultados de ensayos de concretos.

Bedoya, C.; Dzul, L. (2015). El concreto con agregados reciclados como proyecto de sostenibilidad urbana. Revista ingeniería de construcción, 30(2), 99-108. URL: https://dx.doi.org/10.4067/S0718-50732015000200002

Brough, A. R.; Dobson, C.M. (1994). Application of selective 29. Si isotopic enrichment to study of the structure of calcium silicate hydrate (C-SH) gels. Journal of the American Ceramic Society 77(2): 593- 596

Características del Concreto-Resistencia (2009). URL:http://elconcreto.blogspot.pe.

Castro, A.; Ferreira, F. (2016). Efecto de empaquetamiento de las partículas en la durabilidad de los hormigones de alto desempeño. Revista ingeniería de construcción, 31(2),91-104. URL: https://dx.doi.org/10.4067/S0718-50732016000200003

Castro, A.L de; Pandolfelli, VC. (2009). Revisión: conceptos de dispersión y envasado de partículas para la producción de hormigones especiales aplicados en construcción civil. Cerámica, vol.55, n.333, pp.18-32. 
ENGLISH VERSION

Cemento Yura (s.f.). URL: http://www.yura.com.pe

Choobbasti, A.J.; Kutanaei, S.S. (2017). Microstructure characteristics of cement-stabilized sandy soil using nanosilica. Journal of Rock Mechanics and Geotechnical Engineering, Volume 9, Issue 5, 2017, Pages 981-98

Construcción y tecnología en concreto (s.f.). Estructura, URL: http://www.imcyc.com/revistacyt/oct10/tecnologia.htm

Dobson, C. M.; Goberdhan, D.G.C. (1988), 29. Si MAS NMR study of the hydration of tricalcium silicate in the presence of finely divided silica. Journal of Material Science 23: 4108-4111

Du, H.; Pang, S.D. (2019). High performance cement composites with colloidal nano-silica, Construction and Building Materials, Volume 224.

El-Feky, M. S.; Youssef, P.; El-Tair, A. M.; Ibrahim, S.; Serag, M. (2019). Effect of nano silica addition on enhancing the performance of cement composites reinforced with nano cellulose fibers.

Ficha Técnica del aditivo Gaia nanosílice. Cognoscible Tecnologies, (s.f.). Divulgación general.pub -UPV, 2, URL: http://personales.upv.es/fbardisa/Pdf/Gaia\%20nanoproducto .

Fuentes, M.; Zúñiga,A.; Díaz, M.; Rocha, E.; Díaz, S. (2014). Molienda mecánica por alta energía de minerales mexicanos para producir concreto de alto desempeño (CAD). Revista ingeniería de construcción, 29(3), 256-269. URL:https://dx.doi.org/10.4067/S071850732014000300003.

Huang, Q.; Zhu, X.; Liu,D.; Zhao, L.; Zhao, M. (2021).Modification of water absorption and pore structure of high-volume fly ash cement pastes by incorporating nanosilica. Journal of Building Engineering, Volume 33, 101638.

Kotsay, G. (2017). Peculiarities of hydration of Portland cement with synthetic nano-silica. Selected Scientific Papers-Journal of Civil Engineering, 12(2), 101-106.

Lim, S.; Lee,H.S.; Kawashima, S. (2018). Pore structure refinement of cement paste incorporating nanosilica: Study with dual beam scanning electron microscopy/focused ion beam (SEM/FIB), Materials Characterization, Volume 145.

Lincy, V.; Rao, V. V. L. K.; Lakshmy, P. (2018). A study on nanosilica- and microsilica-added concretes under different transport mechanisms. Magazine of Concrete Research, 70(23), 1205-1216.

Mehta, P. K.; Monteiro, P.J.M., (2001). Microstructure, Properties and Materials.

Mendoza, O. (2013). Efecto híbrido de los nanotubos de carbono y la nanosílice sobre las propiedades mineralógicas y mecánicas de morteros de cemento portland, Colombia.

Meng, T.; Yu, H.; Lian, S.; Meng, R. (2020). Effect of nano-SiO 2 on properties and microstructure of polymer modified cementitious materials at different temperatures. Structural Concrete, 21(2), 794-803.

Oliveira, I.R.; Studart, A.R.; Pileggi R.G.; Pandolfelli V.C. (2000). Dispersão e empacotamento de partículas: principios e aplicações em processamento cerámico, São Paulo: Fazendo Arte Editorial, 224p.

Quercia, G.; Spiesz, P.; Hüsken, G.; Brouwers, H. J. H. (2014). SCC modification by use of amorphous nano-silica. Cement and Concrete Composites, 45, 69-81.

Sikora, P.; Rucinska, T.; Dietmar, Stephan; Chung, Sang-Yeop; Abd Elrahman, M. (2020). Evaluating the effects of nanosilica on the material properties of lightweight and ultra-lightweight concrete using image-based approaches, Construction and Building Materials, Volume 264.

Tobón, J. I.; Mendoza Reales, O.; Restrepo, O. J.; Borrachero, M. V.; Payá, J. (2018). Effect of pyrogenic silica and nanosilica on portland cement matrices. Journal of Materials in Civil Engineering, 30(10), 04018266.

Varghese, L.; Rao, V. V. L. K.; Parameswaran, L. (2019). Nanosilica-added concrete: strength and its correlation with time-dependent properties. Proceedings of the Institution of Civil Engineers-Construction Materials, 172(2), 85-94.

Wang, X. F.; Huang, Y. J.; Wu, G. Y.; Fang, C.; Li, D. W.; Han, N. X.; Xing, F. (2018). Effect of nano-SiO 2 on strength, shrinkage and cracking sensitivity of lightweight aggregate concrete. Construction and Building Materials, 175, 115-125.

Zhang, M.; Li, H. (2011). Pore structure and chloride permeability of concrete containing nano-particles for pavement. Construction and Building Materials, 25 (2), pp. 608-616. 